\title{
DEVELOPMENT OF AN OPTIMAL TECHNIQUE FOR RELIABILITY ANALYSIS OF POWER LINE COMMUNICATION SYSTEM FOR SMART GRID REALIZATION
}

\author{
Ganiyu Adedayo Ajenikoko ${ }^{1}$ and Victor Uchenna Nwagbara ${ }^{2}$ \\ 1,2. Department of Electronic and Electrical Engineering, Ladoke Akintola University of Technology, P.M.B, \\ 4000, Ogbomoso, Nigeria. \\ Corresponding Email: ajeedollar@gmail.com
}

\begin{abstract}
Power Line Communication (PLC) is a communication technology that enables transmission of data on a conductor which is also used for electric power transmission. The development of a PLC system presents a significant challenge for the communication engineer due to the unusual channel characteristics that affect high-speed signal transmission since the electric power grid is designed to operate at 50/60 Hz. In addition, the topology of electric power grid network is often very irregular resulting in significant dispersion of the transmitted message signals as it is susceptible to noise and attenuation due to power lines being primarily used for transmission of electricity as a result of its viability for smart grid realization. This study therefore investigates the reliability analysis of PLC for Smart Grid (SG) realization using clipping and Turbo equalizer techniques to reduce the effects of noise and attenuation in narrowband $(9-490 \mathrm{kHz})$ PLC system respectively. This optimal technique is based on the minimum Bit Error Rate (BER) search. The results show that the technique used improves the BER performance of the narrowband PLC system for smart grid realization. The result of this study provides a reliable communication and control for cost efficiency of existing power line infrastructure for Smart Grid.
\end{abstract}

Keywords: PLC, Smart Grid, Attenuation, Clipping, Equalizer, Electric Power Transmission, Bit Error Rate.

DOI: $10.7176 / \mathrm{MTM} / 9-8-01$

Publication date: August 31st 2019

\section{Introduction}

Smart Grid (SG) is a big technological innovation that has the potential to reduce the climate change through a variety of operation and energy measures including the integration of renewable energy resources [1]. SG allows for a two-way communication of data, which helps in real time data collection, monitoring, analysis, control, reduce energy consumption cost and improve overall efficiency of transmission of electric power distribution grid and also reduce the need to build additional infrastructure. The electric power distribution grid is a medium over which fast and reliable communication services can be provided. Communication for SG is a very important aspect and a method that seems a better option to achieve this is PLC [2, 3].

In recent years, increased interest in PLC systems for both commercial and residence applications has resulted in the development of standards for use of the electric power grid as a communication channel conveying messages in addition to power [2]. The type of applications range from simple inexpensive services centered on networked household appliances, where data rates are on the order of kilobits per second, to Internet access via the electrical outlet wall socket, where data rates are on the order of megabits per second. Currently, PLC systems can accommodate high speed networking that includes broadband 
Internet access, voice over-IP, and the interconnectivity of home entertainment devices $[1,3$, $5,6,7]$.

PLC provides the natural upgrade from simple electricity to hybrid and bidirectional electricity and data communication solutions. PLC technologies can either be Narrow-band PLC or Broadband PLC [3]. However, PLC systems are limited due to varying impedance, high attenuation and noise. The noise interference and channel multipath effects are the main impairments to the performance of PLC systems especially when the Signal to Noise Ratio (SNR) is at a decent level [5, 8, 9].

According to Kim et al., (2011), the noise at low frequency band is more energetic than at higher frequencies in broadband PLC systems which results in performance degradation of conventional narrowband PLC systems. The main reason for low performance of conventional narrowband PLC systems is that they use the simple modulation schemes, such as frequency shift keying (FSK) and quadrature phase shift keying (QPSK). Thus, there is the need for better PLC systems that will result in higher performance in order to overcome these limitations. This study presents appropriate channel models for use in the design of PLC systems in order to investigate the effect of PLC on smart grid realization [7]. The noise scenario of power line is shown in Figure 1.

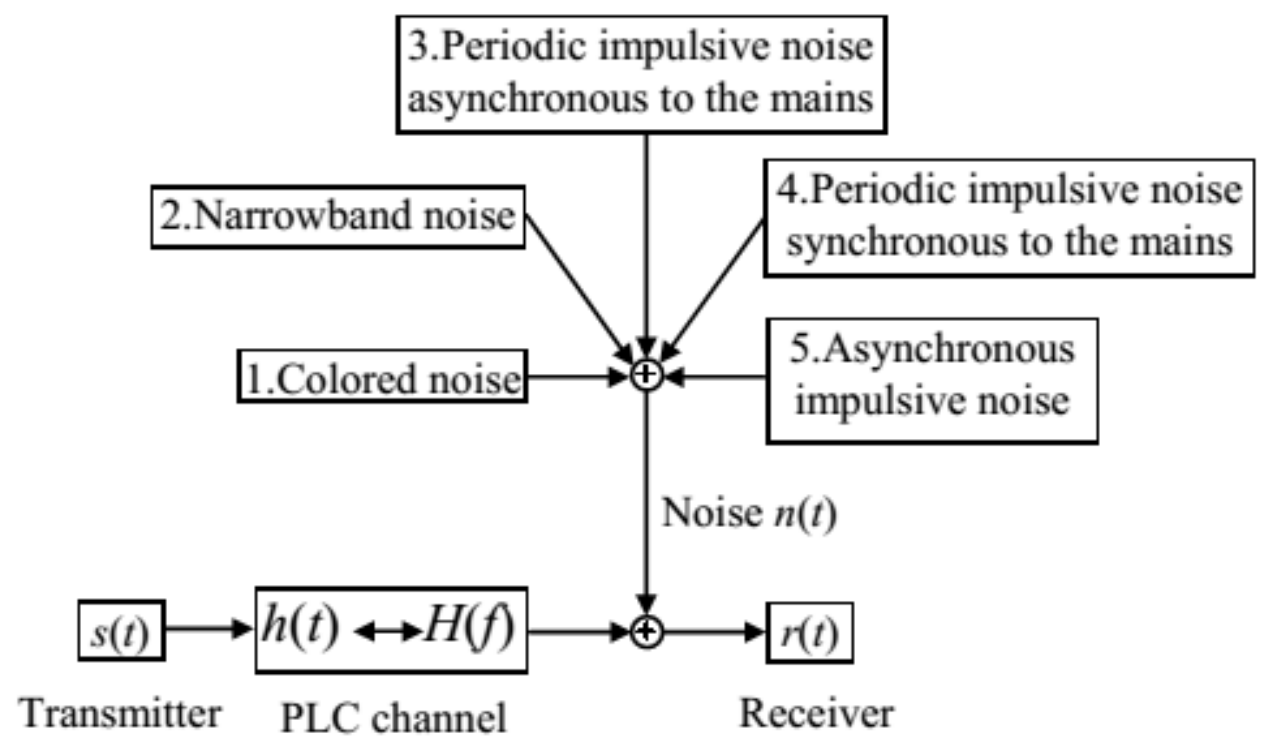

Figure 1: Noise Scenario on Power Lines.

\subsection{Power Line Communication System}

According to Luka et al. (2015) PLC is one of the communication technologies that can be implemented for SG realization. PLC provides communication and control capabilities, using the existing power line network. The use of PLC for SG proves to be cost effective, secure and reliable, as it is implemented by adding a modulated carrier signal to the power cables [9].

In addition Berger et al. (2013) explained that PLC can be differentiated by the operation voltages of power lines. High-Voltage (HV) lines with voltages in range of $110 \mathrm{kV}$ to $380 \mathrm{kV}$ are used for nationwide (Wide Area Network-WAN) power transmission via overhead lines. Medium-Voltage (MV) lines have voltages in the range of $10 \mathrm{kV}$ to $30 \mathrm{kV}$ which are connected to $\mathrm{HV}$ lines through a primary transformer sub-station and are used for power distribution in cities, large industrial regions (Neighbourhood Area Network-NAN). LowVoltage (LV) lines have voltages in the range of $110 \mathrm{~V}$ to $400 \mathrm{~V}$ and are connected to MV lines 
through secondary transformers sub-station, with the Home Area Network (HAN) implemented on LV lines. MV lines are coupled with a PLC repeater to establish a high data rate communication to reduce the effect of heavy attenuation on LV lines [3].

PLC technology can be divided into two; Broadband PLC and Narrowband PLC [10, 11, 12].

i.Broadband Power Line Communication (BPLC): BPLC operates in the high frequency range of $1.8-250 \mathrm{MHz}$ and has data rates up to hundreds of megabits per second, used to provide broadband internet access.

ii.Narrowband Power Line Communication (NBPLC): NBPLC operates in the low frequency range from $3 \mathrm{kHz}$ to $500 \mathrm{kHz}$. NBPLC supports indoor and outdoor communications with LV and MV power lines. NBPLC are capable of data rates of up to 500 kilobits per second. NBPLC is more secured, reliable and cost-effective and it is more preferred for home automation and smart metering.

\subsection{Operation of PLC}

PLC systems operate by imposing a modulated carrier, an Orthogonal Frequency Division Multiplexing (OFDM) signal, on the wiring system. This is due to the fact that the existing power line system is made solely for transmission of $\mathrm{AC}$ power at frequencies of 50 or $60 \mathrm{~Hz}$, making power lines having a limited ability to support higher frequencies. Presently, PLC offers advantages for SG applications, and also has some challenges, which need to be considered to have a proper working PLC model $[13,14]$. The operation network of PLC on smart grid distribution network is shows in Figure 2 [15].

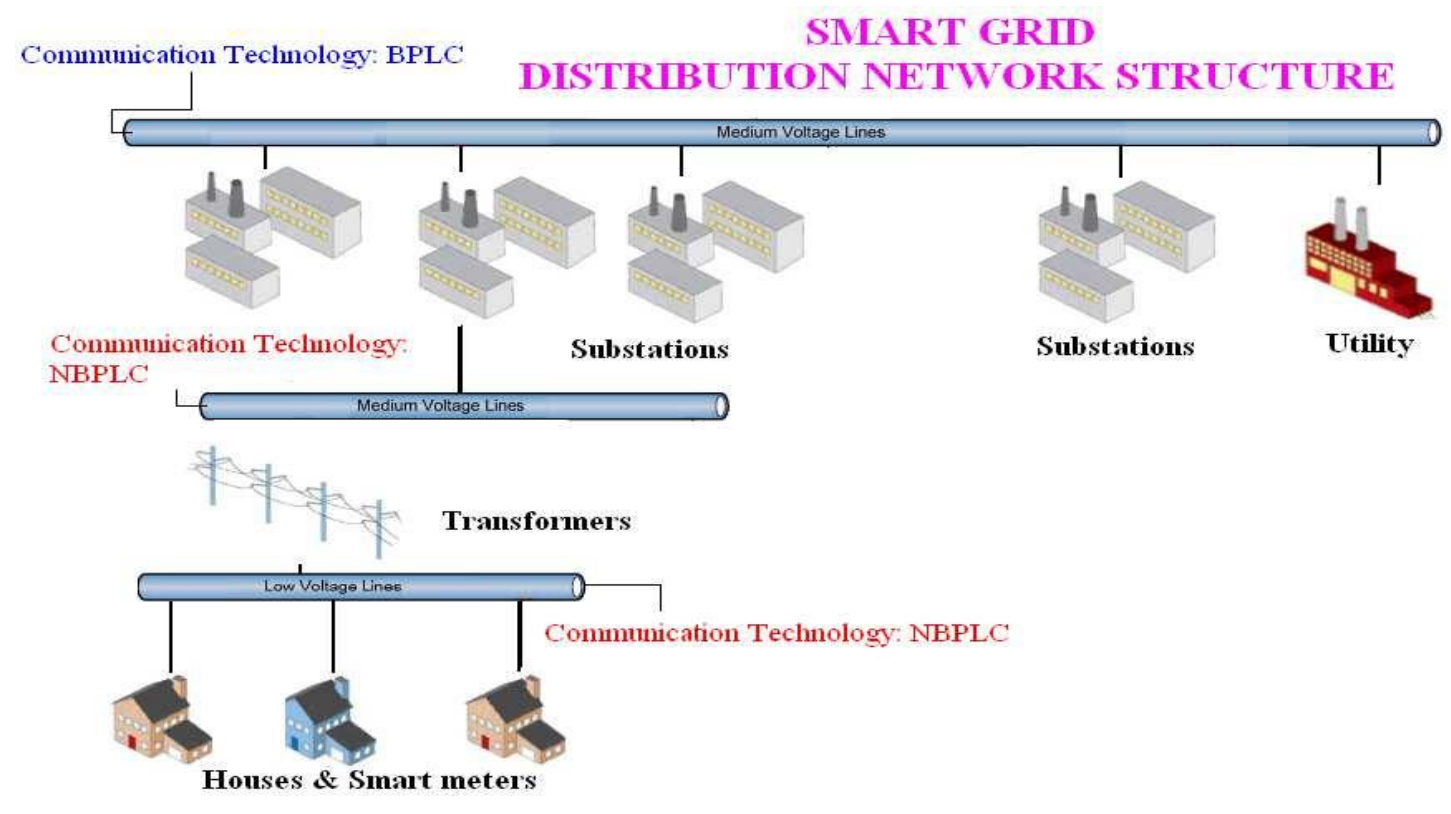

Figure 2: Smart Grid Distribution Network Architecture

\subsection{Application of Power Line Communication on Smart Grid}

The fundamental purpose of Smart Grid is to provide a real time monitoring and control function for an improved efficiency of electricity transmission [15]. The use of PLC for SG has several applications. These applications will be discussed below $[13,14]$.

i. Advanced Metering Infrastructure: PLC enables a two-way communication that can be utilized to exchange information between customer devices and control systems in an AMI. AMI can measure, analyse, and collect energy usage from meters through the use of 
PLC on a real time basis. Although Ultra Narrowband PLC technology has low data rate, UNB-PLC is being used for remote meter reading and can cover large distances up to $150 \mathrm{~km}$.

ii.Demand Side Management (DSM): An application of DSM is Demand Response (DR). DR answers the demands of various energy requests. DR helps to reduce peak demand and enable consumers to monitor energy consumption. DR controls peak, power conditions, and increases power system efficiency by adopting a real time pricing scheme. The DR system is implemented through the BB-PLC.

iii.In-home Environment: The Home Energy Management System (HEMS) offers advantages to the grid, such as reliability and reduction of peak demand due to the continuous monitoring and reporting of energy demands to the grid.

iv.Fault Detection: High-Voltage (HV) lines can be used to remotely detect faults such as short-circuited insulators or cable outbursts. Traffic losses, signalling errors can be prevented through remote fault detection by PLC.

\subsection{Advantages of PLC in smart grid}

PLC offers some advantages for smart grid applications. Some of the advantages are [12]:

i. A wide area is covered due to the existing power line infrastructure, thereby providing mobility for SG applications.

ii. It is relatively cheap as there is no need for additional cabling.

iii. PLC systems are flexible and can also cover long range communication.

iv. PLC systems provide a stable communication system for SG applications.

v. Installation of PLC systems is easy for indoor implementations.

\subsection{Materials and Methods}

In this study, a PLC model for SG was developed. Clipping and Turbo equalizer techniques were used to analysis the reliability of PLC for SG realization in order to reduce the effects of noise and attenuation in narrowband PLC system. Clipping technique was used to solve the problem of noise in PLC system. Clipping is a method in which the amplitude of the received signal is cut off over the threshold level without its phase change. In this approach a clipping block is added to the front-end of the Orthogonal Frequency Division Multiplexing (OFDM) receiver before demodulating. OFDM is a modulation technique on narrowband LV PLC, due to its robustness to multipath, frequency selective fading and different kinds of interference. In contrast to the single carrier systems, OFDM systems present better performance in impulsive noisy environments.

The study employed Binary Phase Shift Keying (BPSK), Quadrature Phase Shift Keying (QPSK) and 16 Quadrature Amplitude Modulation (16QAM) as a modulation scheme and simulate without and with clipping and with no channel coding and with convolutional code. The received signal with clipping can be expressed as:

$$
y_{n}=\left\{\begin{array}{c|c}
r_{n} & \left|r_{n}\right| \leq T_{C} \\
T_{C} e^{j \arg \left(r_{n}\right)} \mid & \left|r_{n}\right|>T_{C}
\end{array}, n=0,1, \ldots, N-1\right.
$$

Where; $T_{C}$ denote clipping threshold, $N$ is the number of power line branches, $e^{j \arg \left(r_{n}\right)}$ is attenuation term

Turbo Equalizer technique was also used to solve channel attenuation in the PLC system. This technique works by jointly performing Maximum Posterior (MAP) detection or 
near-MAP detection for the inter-symbol interference channel (inner code) and soft-decoding the channel code (outer code) through an iterative process.

The Bar Error Rate of an OFDM system with N subcarriers is given as:

$$
B E R_{\text {avg }} \leq \frac{1}{\log _{2} M}\left(1-\frac{\sum_{k=1}^{N}\left(1-P_{k, \sqrt{M}}\right)^{2}}{N}\right)
$$

where

$$
P_{k, \sqrt{M}}=2\left(1-\frac{1}{\sqrt{M}}\right) Q\left(\sqrt{\frac{3 H_{k}^{2} E_{a v}}{(M-1) N_{0}}}\right)
$$

where; $H_{k}$ is the channel transfer function of $k^{\text {th }}$ sub channel

\subsection{System Model}

The PLC system model used for this study is depicted in Figure 3. The input data is modulated by channel coding at the convolution encoder, which neutralizes the effect of channel fading. Then the signal passes through the OFDM modulator, with the input signal being imposed with an OFDM signal and then modulated to increase the frequency of the signal to be transmitted on the power line. As power lines are only capable of transmitting analog signals, the OFDM signal which is digital signal is converted to analog signal by the digital-to-analog converter to be transmitted via the power lines. The received signal is then converted back to digital by the analog-to-digital converter. This received signal has been affected by noise, attenuation. This noise and channel attenuation are reduced through the clipping and equalizer block. Then the received signal is then demodulated and the original data is recovered.

In the study, the size of data frame and power line length are 3052 and 318 samples respectively, and 35 packets are transmitted. Simulation is performed on the number of power line branches. The length of main line is fixed as $40 \mathrm{~m}$ and length of the branches are determined randomly in the range of 2 to $10 \mathrm{~m}$. To enhance the performance of PLC technology, convergent network architecture with integration of Ethernet-based networks was considered. This convergence was achieved through a shared common layer, the Data Link or Physical Layer that enables interconnectivity. The integration of Ethernet network using the TCP/IP protocol to form a convergent network was also used to mitigate attenuation. 


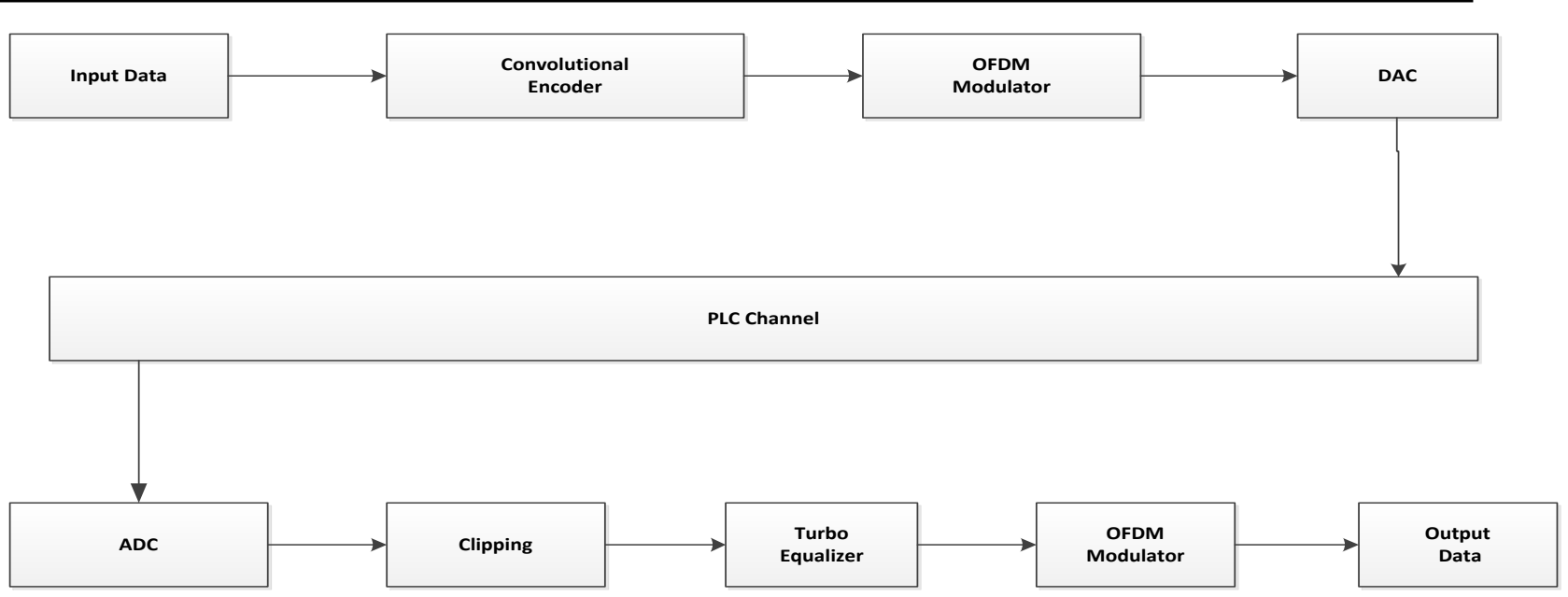

Figure 3: PLC system model

\subsection{Results and Discussion}

The analysis of results for reliability of Power Line Communication System for Smart Grid realization is presented in Figure 4 to Figure 7. The result of noise reduction was presented without and with clipping and with no channel coding and with convolutional code. While the attenuation of PLC was presented with frequencies channel responses of power line branches of 3 and 5.

Figure 4 shows the results of BER for PLC system without and with clipping with no coding. For PLC system without clipping, the BER values for BPSK are 0.2, 0.06, 0.03, 0.006, 0.003, 0.001 and 0.0003. The QPSK have the BER values of 0.3, 0.1, 0.05, 0.01, 0.005, 0.005 and 0.0005. 16QAM have the BER values of 0.5, 0.3, 0.1, 0.06, 0.02, 0.007 and 0.002 . The result of BER for PLC system with clipping shows that BPSK have BER values of 0.3, 0.05, 0.02, 0.005, 0.004, 0.002 and 0.0002. The BER values for QPSK are 0.3, 0.2, 0.06, 0.02, 0.006, 0.006 and 0.0007. In addition, the BER values of 16QAM are 0.5, 0.3, 0.2, 0.07, 0.03, 0.008 and 0.003. In general, the results show that BPSK, QPSK and 16QAM illustrate $25 \mathrm{~dB}$, $28 \mathrm{~dB}$ and $32 \mathrm{~dB}$ BER performance at 0.01 with no clipping respectively. With clipping scheme, the performance of all the modulation increases from 0.5 to $0.6 \mathrm{~dB}$. The results therefore confirm that clipping improves the system performance slightly.

Figure 5 shows the results of BER for PLC system without and with clipping with convolutional code. For the PLC system without clipping, the BER values for BPSK are 0.3, $0.05,0.02,0.006,0.0006,0.00008$ and 0.00004 . The QPSK have the BER values of $0.5,0.2$, $0.05,0.009,0.001,0.0005$ and 0.0002. 16QAM have the BER values of 0.6, 0.5, 0.2, 0.07, $0.009,0.001$ and 0.0007 . The results of BER for PLC system with clipping with convolutional code indicate that BPSK have BER values of 0.2, 0.06, 0.03, 0.005, 0.0005, 0.00009 and 0.00003. The BER values for QPSK are 0.5, 0.3, 0.06, 0.02, 0.002, 0.0004 and 0.0003 . In addition, the BER values of 16QAM are 0.6, 0.5, 0.3, 0.08, 0.02, 0.002 and 0.0008 . In general, at BER of 0.001 , the overall system performance are improved approximately to $7 \mathrm{~dB}$ and there is 0.4 to $0.8 \mathrm{~dB}$ additional improvement in terms of the effect of clipping. Therefore, BER performance for BPSK, QPSK, and 16QAM are $18.5 \mathrm{~dB}, 22.3 \mathrm{~dB}$ and $26.6 \mathrm{~dB}$ respectively.

Figure 6 and Figure 7 illustrate channel responses at power line branches of 3 and 5 respectively. The results show that PLC channels suffer from frequency selective fading. It 
was confirmed that the magnitude of the channel is attenuated as the frequency increases. In addition, as the number of branches increase, the channel state becomes poor due to occurrence of the reflection between devices and branch lines of the PLC system. Therefore, the reflection seriously affects the PLC channel in terms of Inter-Symbol-Interference (ISI).

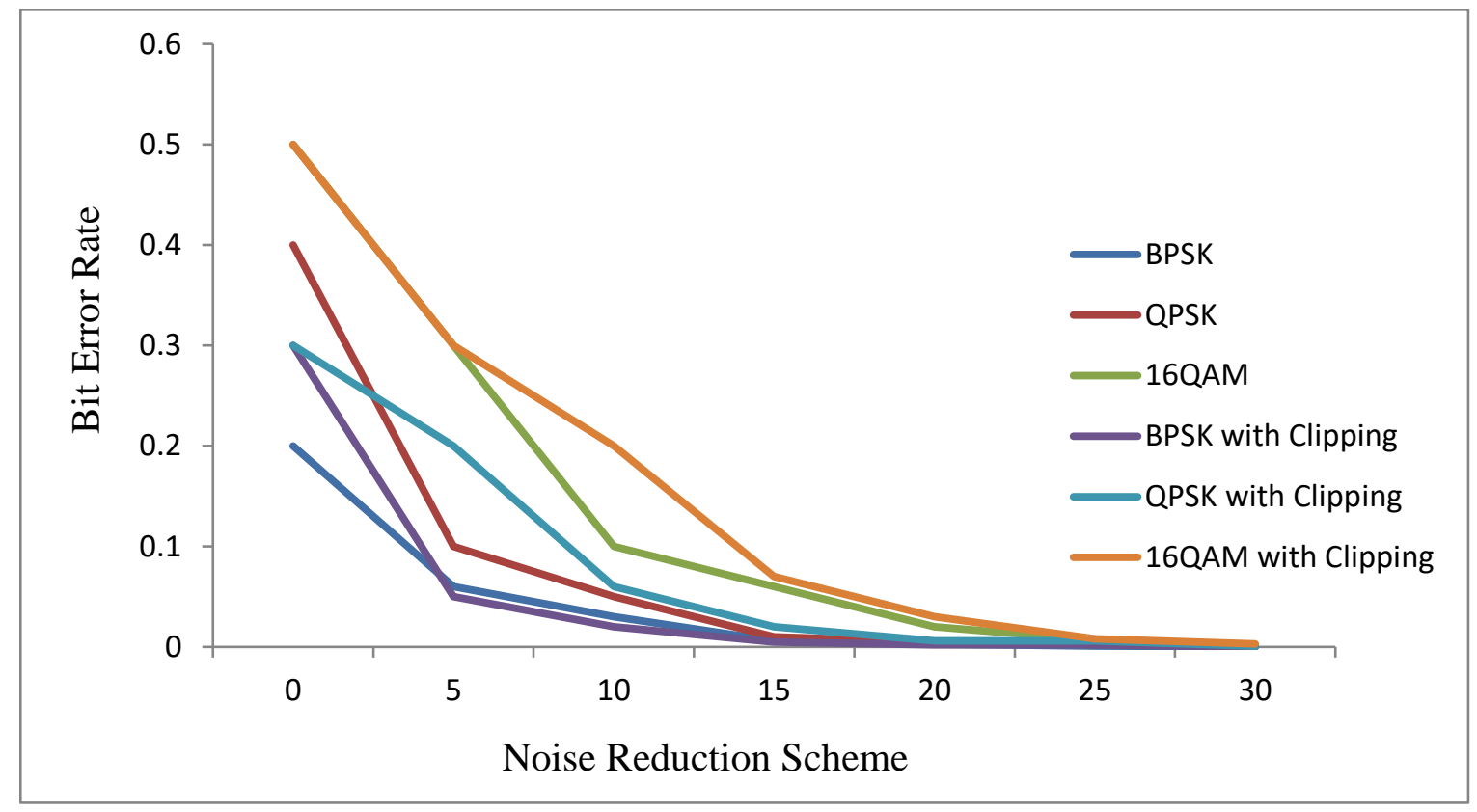

Figure 4: Performance of Bit Error Rate with Noise Reduction Scheme in PLC Channel without coding

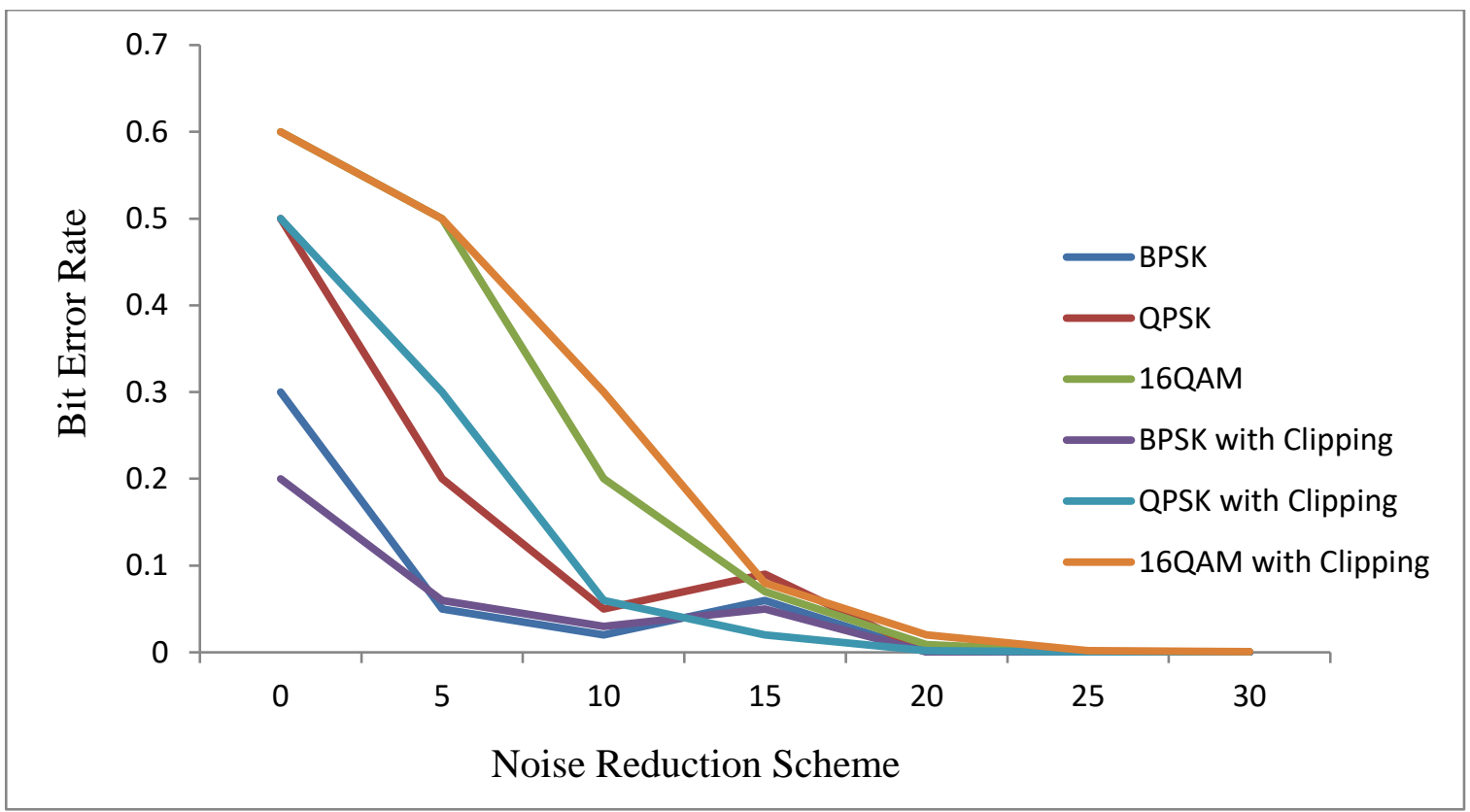

Figure 5: Performance of Bit Error Rate with Noise Reduction Scheme in PLC Channel with Convolutional code 


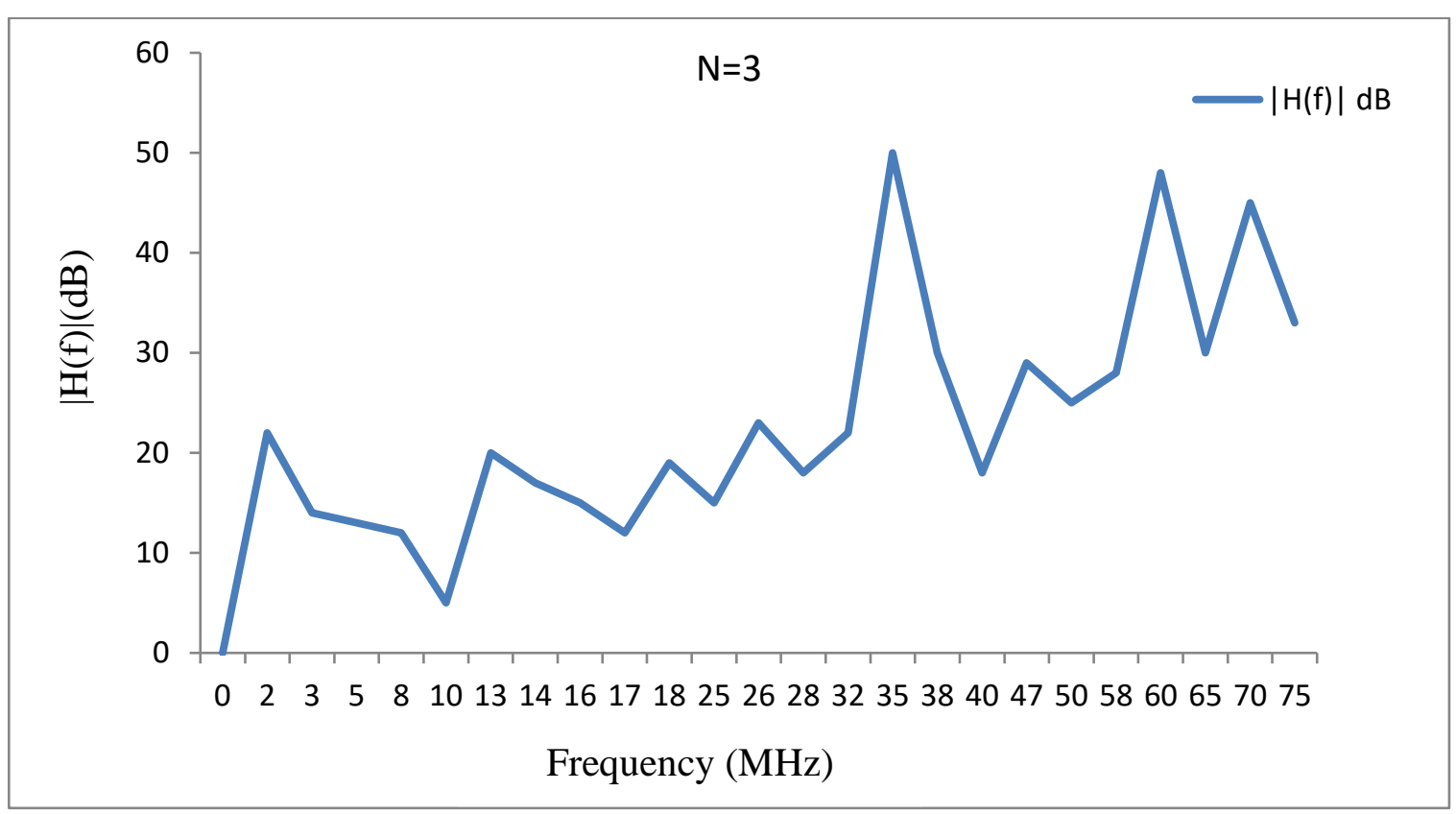

Figure 6: Channel Response at Power Line Branch of 3

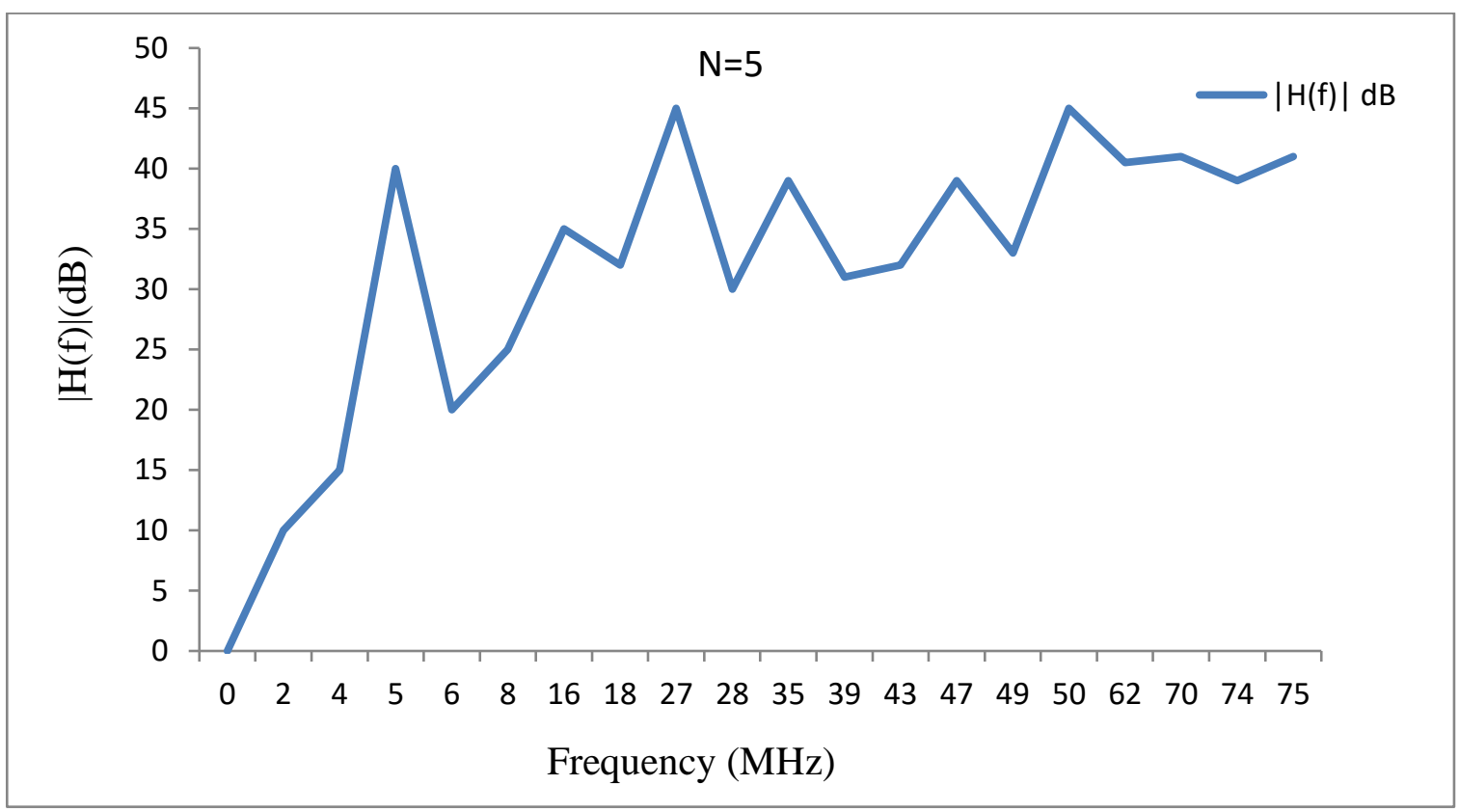

Figure 7: Channel Response at Power Line Branch of 5

\section{Conclusion}

This study has analysed the reliability of Power Line Communication System for Smart Grid Realization in order to reduce the effects of noise and attenuation in narrowband PLC system using Clipping and Turbo equalizer techniques. PLC has been seen as a solution for the realization of Smart Grid. It is easy to build as it uses existing power line infrastructures. 
The study revealed that the clipping scheme performed better than conventional PLC system. There exists an optimal threshold value for clipping nonlinearity, corresponding to a Noise Reduction Scheme (NRS) value, at which the BER is minimized. In addition, the result indicated that PLC channels suffered from frequency selective fading and that the magnitude of the channel attenuated as the frequency increased. Therefore, the clipping scheme is good for mitigating impulsive noise in PLC system for smart grid.

\section{References}

[1] Akarte, V., Punse, N. and Dhanorkar, A. (2014). Power line communication systems. International Journal of Innovation, Electrical and Electronic Instrumentation Control Engineering, 2(1): 709-713.

[2] Baimel, D., Tapuchi, S. and Baimel, N. (2016). Smart grid communication technologies. Journal of Power and Energy Engineering, 4(8): 1-8.

[3] Berger, L. T., Schwager, A. and Escudero-Garzás, J. J. (2013). power line communications for smart grid applications. Journal of Electrical and Computer Engineering, 2013, Article ID 712376; 1-16.

[4] Di-Bert, L., D'Alessandro, S. and Tonello, A. M. (2013). Enhancements of G3-PLC technology for smart-home/building applications", Journal of Electrical and Computer Engineering, 2013, Article ID 746763, 1-11.

[5] Fariba, A. (2015). Viability of power line communication for smart grid realization. Master Thesis submitted to the Department of Electrical and Computer Engineering, Queen's University Kingston, Ontario, Canada, 1-92.

[6] Galli, S., Scaglione, A. and Wang, Z. (2011). For the grid and through the grid: the role of power line communications in the smart grid. To Appear in the Proceedings of the IEEE - June 2011; 1-26.

[7] Kim, Y. C., Bae, J. N. and Kim, J. Y. (2011). Performance of power line communication systems with noise reduction scheme for smart grid applications. IEEE Transactions on Consumer Electronics, Vol. 57, No. 1, pp. 46-52, February 2011.

[8] Kim, Y., Bae, J. N. and Kim, J. Y. (2011). Performance of power line communication systems with noise reduction scheme for smart grid applications. IEEE Transactions on Consumer Electronics, 57(1): 46-52.

[9] Luka, M. K., Pallam, S. ,W., Thuku I. T. and Etuk, U. U. (2015). Narrowband power line communication for smart grid. International Journal of Scientific and Engineering Research 6(7): 1244-1251.

[10] Mario, C. and Salvatore, T. (2014). A flexible approach for smart management of transmissions in power line communications. Hindawi Publishing Corporation Journal of Computer Networks and Communications, 2014: 1-10.

[11] Mehdi, K., Nasser, H. and Taleb, M. (2011). Performance evaluation of a narrowband power line communication for smart grid with noise reduction technique. IEEE Transactions on Consumer Electronics, 57(4): 1598-1606.

[12] Nguyen, T. V., Petit, P., Aillerie, M., Charles, J. P. and Le, Q. T. (2015). Power line communication system for grid distributed renewable energy. Journal of Fundamentals of Renewable Energy and Applications, 5(3): 1-6.

[13] Ünsal, D. B. and Yalçınöz, T. (2015). Applications of new power line communication model for smart grids. International Journal of Computer and Electrical Engineering, 7(3): 168-178 
[14] Xie, K. and Li, J. (2010). Precoded turbo equalizer for power line communication systems. ArXiv: 1004.0180.

[15] Yigit, M., Gungor, V. C., Tuna, G., Rangoussi, M. and Fadel, E. (2014). Power line communication technologies for smart grid applications: A review of advances and challenges", Computer Networks, 70; 366-383. 